\title{
ESTUDOS DE BIOLOGIA DA PESCA DE LAGOSTAS NO CEARÁ - DADOS DE $1970^{(1)}$
}

\author{
Melquiades Pinto Paiva - Raimundo Saraiva da Costa
}

\author{
Laboratório de Ciências do Mar \\ Universidade Federal do Ceará \\ Fortaleza - Ceará - Brasil
}

Com o presente trabalho, o Laboratório de Ciências do Mar da Universidade Federal do Ceará dá prosseguimento à série de estudos sobre a biologia da pesca de lagostas no Ceará (Paiva \& Silva, 1962 ; Paiva \& Costa, 1963, $1964,1965,1966,1967,1968,1969$ e 1970)

A partir do corrente ano, as pescarias de lagostas realizadas na costa cearense passaram a ser controladas através de mapas de bordo, tornados de uso obrigatório pela Superintendência do Desenvolvimento da Pesç Alguns dados constantes dos mesmos foram utilizados no presente trabalho, tendo em vista, principaimente, o conhecimento da densidade relativa das lagostas.

Durante o ano de 1970 , a exportação brasileira de caudas congeladas de lagostas aicançou o total de 2.793 toneladas métricas. tendo sido realizada pelos portos de Fortaleza, Recife, Cabedêlo, Rio de Janeiro e Uruguaiana, em ordem decrescente. Somente pelo porto de Fortaleza foram exportadas 2.036 toneladas métricas de caudas congeladas de lagostas, que representaram $72,9 \%$ da exportação nacional do produto, no referido ano.

A área explorada pelas empresas lagosteiras sediadas em Fortaleza, durante o ano de 1970 , praticamente se restringiu à plataforma continental que corresponde ao Estado do Ceará.

\section{MATERIAL}

O material em que se baseia este trabalho foi capturado em frente ao município de Fortaleza, havendo sido desembarcado na praia de Mucuripe. As amostras correspondem a 210

(1) - Trabalho realizado em decorrência de convênios celebrados com a Superintendência do Desenvolvimento da Pesca (SUDEPE) e a Superintendência cio Desenvolvimento do Nordeste (SUDENE). dias de pescarias, realizadas no período de 2 de janeiro a 30 de dezembro de 1970 , por pequenos botes motorizados, que pescaram somente com manzuás, iscados principalmente com peixes marinhos e, em menor escala, com peixes de água doce. Foram amostrados 10.500 indivíduos (tabela I) . Também, foram utilizados os dados do esforço de pesca e de lagostas capturadas, de pescarias controladas através de mapas de bordo, na mesma área e período acima referidos (tabela VII) .

\section{MÉTODO}

Em cada amostra tomamos um total de 50 indivíduos, retirados ao acaso, dos desembarques de lagostas em Mucuripe.

Para cada indivíduo amostrado anotamos a espécie, o sexo, o comprimento total e também se estava ou não em processo de muda ou de reprodução. Com referência a este último, apenas as fêmeas foram consideradas.

Levamos em consideração, exclusivamente, os caracteres sexuais externos, para o registro dos sexos dos indivíduos amostrados.

A medição do comprimento total foi feita no plano de simetria e sobre o dorso do corpo, a partir da margem anterior do entalhe formado pelos espinhos rostrais até a extremicade posterior do telso, estando o animal completamente estendido sobre uma superfície plana. Nas medições utilizamos paquímetro de aço capaz de registrar frações centesimais do centímetro.

Quando uma lagosta se apre: nntava com carapaça fendida, se desprendendo do corpo, ou quando esta estava sem rigidez, por ter havido muda recente, o animal era considerado como em processo de muda.

Todas as fêmeas com espermoteca integra, ovadas ou com restos de espermoteca, foram consideradas como em processo de reprodução. 
T A B E L A I

Numero de amostras e de individuos amostrados em cada trimestre, bem como as frequéncias absolutas $c$ relativas das espécies Panulirus argus (Latr.) e Panulirus laevicauda (Latr.), em cada conjunto trimestral de amostras de lagostas desembarcadas em Mucuripe (Fortaleza - Ceará -.. Brasil), durante o período de janeiro a dezembro de 1970 .

\begin{tabular}{|c|c|c|c|c|c|c|}
\hline \multirow{3}{*}{ Trimestres } & \multirow{3}{*}{$\begin{array}{l}\text { Número } \\
\text { de } \\
\text { amostras }\end{array}$} & \multirow{3}{*}{$\begin{array}{l}\text { Indivíduos } \\
\text { amiostrados }\end{array}$} & \multicolumn{4}{|c|}{ Espécies } \\
\hline & & & \multicolumn{2}{|c|}{ Panulirus argus } & \multicolumn{2}{|c|}{ Panulirus laevicauda } \\
\hline & & & n.o & $\%$ & $\mathrm{n} \cdot{ }^{\circ}$ & $\%$ \\
\hline $\begin{array}{c}\text { I } \\
\text { II } \\
\text { III } \\
\text { IV } \\
\end{array}$ & $\begin{array}{l}39 \\
56 \\
60 \\
55\end{array}$ & $\begin{array}{l}1.950 \\
2.800 \\
3.000 \\
2.750\end{array}$ & $\begin{array}{l}1.195 \\
1.574 \\
1.920 \\
1.070 \\
\end{array}$ & $\begin{array}{l}61,3 \\
56,2 \\
64,0 \\
39,0 \\
\end{array}$ & $\begin{array}{r}755 \\
1.226 \\
1.080 \\
1.680\end{array}$ & $\begin{array}{l}38,7 \\
43,8 \\
36,0 \\
61,0\end{array}$ \\
\hline Total & 210 & 10.500 & 5.759 & 54,8 & 4.741 & 45,2 \\
\hline
\end{tabular}

T A B E L A I I

Freqüências absolutas dos individuos da espécie Panulirus argus (Latr.) por trimestres, sexos e classes de comprimento total, bem como as frequiencias absolutas e relativas destes no total de cada trimestre e ano, por sexos e classes de comprimento total, nas amostras de lagostas desembarcadas em Mucuripe (Fortaleza Ceará - Brasil), durante o periodo de janeiro a dezembro de 1970 .

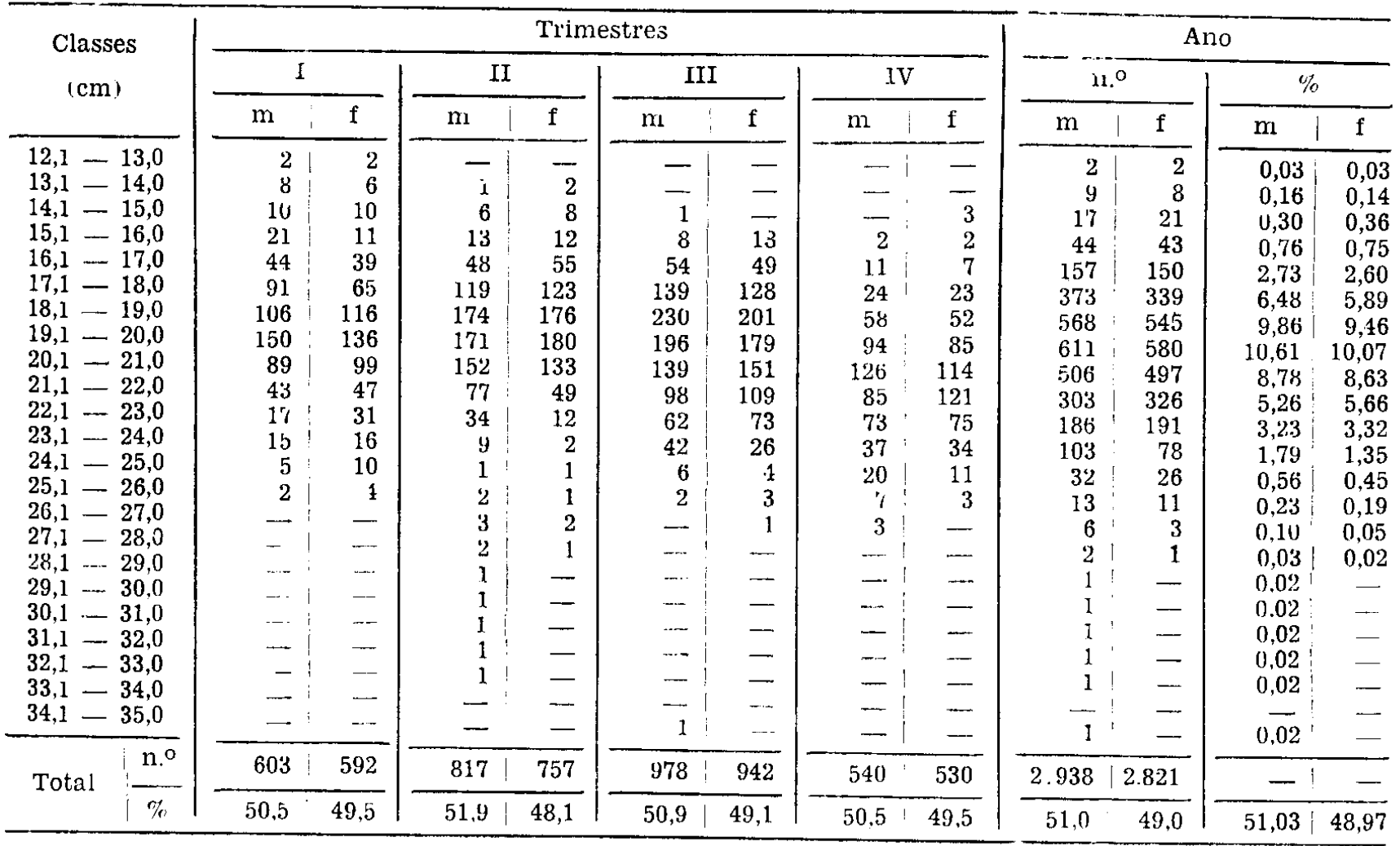

As diferenças verificadas entre as fre. quências absolutas de machos e fêmeas da mesma espécie, no total global amostrado, em cada um dos conjuntos trimestrais de amostras, bem como nas diversas classes de comprimento total da amostragem anual, foram submetidas ao teste do $x^{2}$. Também, empregamos o teste de homogeneidade. A probabilidade de 0,05 foi escolhida como sendo o nível de significância.
() esforço de pesca controlado se baseia nas capturas de lagostas de várias embarcações da frota lagosteira, que operaram com um inconstante número de manzuás por viagem, em frente ao município de Fortaleza.

A partir dos dados do esforço de pesca controlado e do número de lagostas capturadas por espécie e no total, calculamos os índices de densidade ralativa (lagóstas capturadas por manzuá/dia) para cada espécie e 
I A B E L A I I I

Frequéncias absolutas dos individuos da. especic Panulirus la€vicauda (Latr.) por trimestres, sexos e classes de comprimento total, bem como as freqüencias absolutas $\mathrm{e}$ relativas destes no total de cada trimestre e ano, por sixus e classes de comprimento total, nas amostras de lagostas desembarcadas em Mucuripe (Fortaleza - Ceará - Brasil), durante o periodo de janeiro a dezembro de 1970.

\begin{tabular}{|c|c|c|c|c|c|c|c|c|c|c|c|c|c|}
\hline \multirow{3}{*}{\multicolumn{2}{|c|}{$\begin{array}{l}\text { Cilasses } \\
(\mathrm{cm})\end{array}$}} & \multicolumn{8}{|c|}{ Trimestres } & \multicolumn{4}{|c|}{ Ano } \\
\hline & & \multicolumn{2}{|c|}{ I. } & \multicolumn{2}{|c|}{ II } & \multicolumn{2}{|c|}{ III } & \multicolumn{2}{|c|}{ IV } & \multicolumn{2}{|c|}{$\mathrm{n} .{ }^{\circ}$} & \multicolumn{2}{|c|}{$\%$} \\
\hline & & $\mathrm{m}$ & $\mathrm{f}$ & $\mathrm{m}$ & $\mathrm{f}$ & $\mathrm{n}$ & $\mathrm{f}$ & $\mathrm{m}$ & $\mathrm{f}$ & $\mathrm{m}$ & $\mathrm{f}$ & $\mathrm{m}$ & f \\
\hline $12,1-$ & 13,0 & - & $\ldots$ & $\cdots$ & - & -.. & 1 & $\ldots$ & - & $\ldots !$ & $i$ & - & 0,02 \\
\hline 13,1 & 14,0 & $\ldots$ & $\ldots$ & $\ldots$ & -. & 1 & 2 & 2 & $\ldots$ & 3 & 2 & 0,06 & 0,04 \\
\hline $14,1 \quad-$ & 15,0 & 12 & 5 & 7 & 7 & 9 & 9 & 9 & 3 & 37 & 24 & 0,78 & 0,51 \\
\hline 15,1 & 16,0 & 28 & 8 & 50 & 27 & 47 & 35 & 32 & 14 & 157 & 84 & 3,31 & 1,77 \\
\hline 16,1 & 17,0 & 55 & 26 & 135 & 93 & 86 & $5 \overline{5}$ & 116 & 61 & 392 & 235 & 8,27 & 4,96 \\
\hline $17.1 \quad \ldots$ & 18,0 & 113 & 70 & 202 & 206 & 150 & 90 & 235 & 206 & 700 & 572 & 14,77 & 12,07 \\
\hline $18,1 \ldots$ & 19,0 & 145 & 82 & 142 & 180 & 151 & 137 & 300 & 262 & 738 & 661 & 15,57 & 13,94 \\
\hline $19,1 \quad \ldots$ & 20,0 & 72 & 57 & 57 & 66 & 86 & 79 & 146 & 147 & 361 & 349 & 7,61 & 7,36 \\
\hline 20,1 & 21,0 & 24 & 22 & 17 & 10 & 41 & 43 & 60 & 53 & 142 & 128 & 3,00 & 2,70 \\
\hline $21,1 \quad-$ & 22,0 & 8 & 13 & 5 & 9 & 13 & 20 & 9 & 11 & 35 & 53 & 0,74 & 1,12 \\
\hline $22,1 \quad \ldots$ & 23,0 & 5 & 5 & 4 & 7 & 11 & 11 & 1 & 4 & 21 & 27 & 0,44 & 0,57 \\
\hline $23,1 \quad-$ & 24,0 & 1 & 2 & $\ldots$ & 1 & 1 & 1 & 1 & 3 & 3 & 7 & 0,06 & 0,15 \\
\hline $24,1 \quad-$ & 25,0 & $\ldots$ & - & -- & -- & -- & 一 & 2 & 2 & 2 & 2 & 0,04 & 0,04 \\
\hline $25,1 \ldots$ & 26,0 & $\ldots$ & 2 & $\longrightarrow$ & - & - & 1 & - & - & - & 3 & - & 0,00 \\
\hline $26,1-$ & 27,0 & - & - & - & - & - & - & 1 & - & 1 & - & 0,02 & \\
\hline $27.1-$ & 28,0 & - & - & - & 1 & - & - & - & - & - & 1 & - & 0,02 \\
\hline \multirow{2}{*}{ Total } & $n .^{\circ}$ & 463 & 292 & 619 & 607 & 596 & 484 & 914 & 766 & 2.592 & 2.149 & -1 & - \\
\hline & $\%$ & 61,3 & 38,7 & 50,5 & 49,5 & 55,2 & 44,2 & 54,4 & 45,6 & 54,7 & 45,3 & 54,67 & 45,33 \\
\hline
\end{tabular}

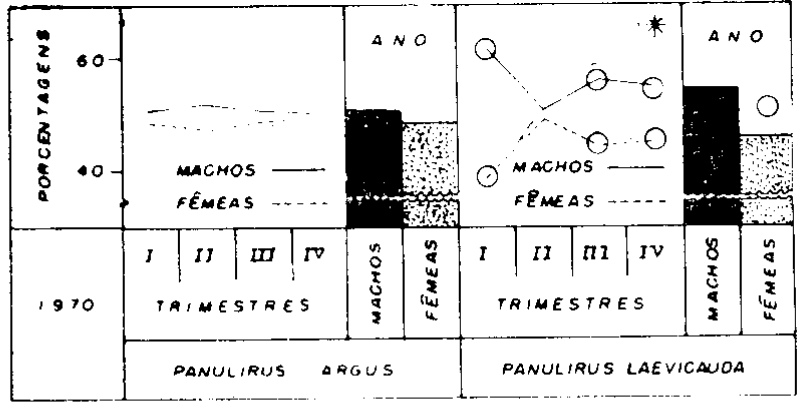

Figura 1 - Freqüencias relativas de machos c fèmeas das espécies Panulirus argus (Latr.) e $P a$ nulirus laevicauda (Latr.), em relação aos totais dos indivíduos amostrados de cada espécie, em cacia conjunto trimestral de amostras, bem como em relação aos totais dos indivíduos amostrados de cada espécie, no conjunto geral das amotras. As diferencas assinaladas por círculos são estatisticamente significativas. O sinal * indica que o correspondentc teste de homogeneidade deu resultado estatisticamente significativo. A probabilidade de 0,05 fo: tomada como nivel de significância.

para ambas em conjunto, correspondendo aos trimestres e ao ano estudado.

\section{DISCUSSÃO E CONCLUSÕES}

Somente as espécies Panulirus argus (Latr.) e Panulirus laevicauda (Latr.) foram atingidas pelas pescarias de lagostas realizadas em águas costeiras fronteiriças ao município de Fortaleza, durante o ano de 1970 (tabela I) .
As amostragens atingiram um total global de 10.500 indivíduos, sendo que 54,8\% correspondem à espécie Panulirus argus e $45,2 \%$ à espécie Panulirus laevicauda.

Quando comparamos os dados dos trabalhos anteriores da série, com os que acabamos de analisar, observamos que, na área em estudo. houve uma sensível redução da participação relativa da espécie Panulirus argus e uma crescente participação relativa da espécie $P a$ nulirus laevicauda nas amostras.

No total das amostras, para ambas as espécies, verificamos terem sido os machos mais atingidos pela pesca do que as fêmeas (tabelas II e III ; figura 1). A diferença constatada entre os totais globais de ambos os sexos, é estatisticamente insignificante na es-

T A B E L A I V

Frecuencias absolutas dos individuos capturados em processo de muda, pertencentes às espécies Panulirus argus (Latr.) e Panulirus laevicauda (Latr.), nas amostras de lagostas desembarcadas em Mucuripe (Fortaleza - Ceará - Brasil), durante o periodo de janeiro a dezembro de 1970 .

\begin{tabular}{|c|c|c|c|c|}
\hline \multirow[t]{2}{*}{ Trimestres } & \multicolumn{2}{|c|}{$\begin{array}{l}\text { Panulirus } \\
\text { argus }\end{array}$} & \multicolumn{2}{|c|}{$\begin{array}{l}\text { Panulirus } \\
\text { iaevicauda }\end{array}$} \\
\hline & machos & fêmeas & machos & fêmeas \\
\hline I & 2 & 6 & - & - \\
\hline II & 20 & 11 & 7 & 3 \\
\hline $\begin{array}{l}\text { III } \\
\text { IV }\end{array}$ & $\begin{array}{l}15 \\
10\end{array}$ & $\begin{array}{l}18 \\
14\end{array}$ & 4 & 1 \\
\hline Total & 47 & 49 & 11 & 4 \\
\hline
\end{tabular}




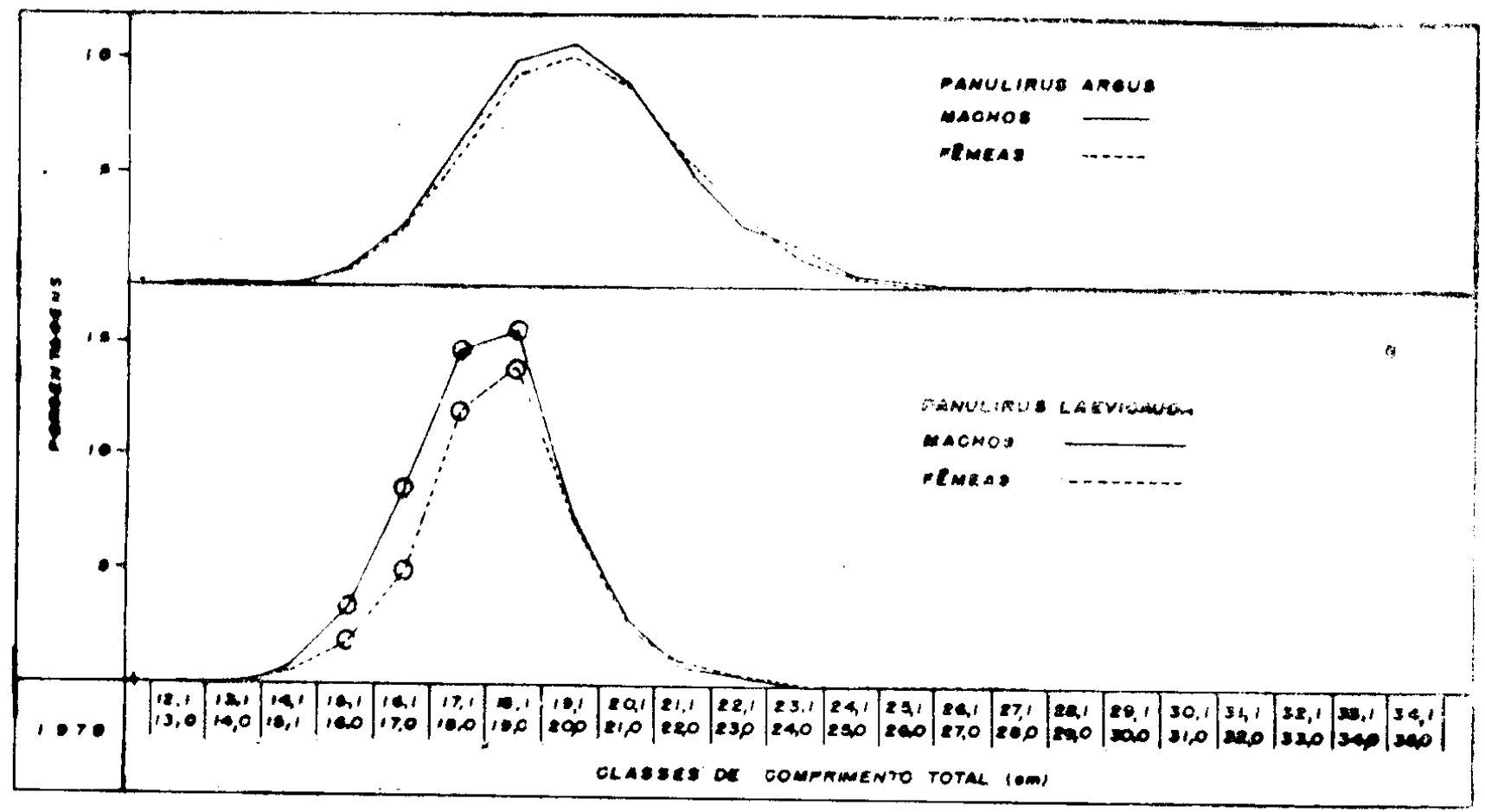

Figura 2 - Freqüencias relativas de machos e fêmeas das espécies Panulirus argus (Latr.) e Panulirus lacvicauda (Latr.), em relaçāo aos totais dos indivíduos amostrados de cada espécie, no conjunto geral dás amostras, e distribuídas por classes de comprimento total. As diferenças assinaladas por círculos são estatisticamente significativas. O sinal * indica que o corr espondente teste de homogeneidade deu resultado estatisticamente significativo. A probabilidade de 0,05 foi tomada como nível de significância.

'I A B E L A V

Freqüèncias de fêmeas de Panulirus argus (Latr.), capturadas em processo de reproduçäo, bem como em cada uma de suas etapas, por trimestres e classes de comprimento total, nas amostras de lagostas desembarcadas em Mucuripe (Fortaleza - Ceará - Brasil), durante o período de janeiro a dezembro de 1970 .

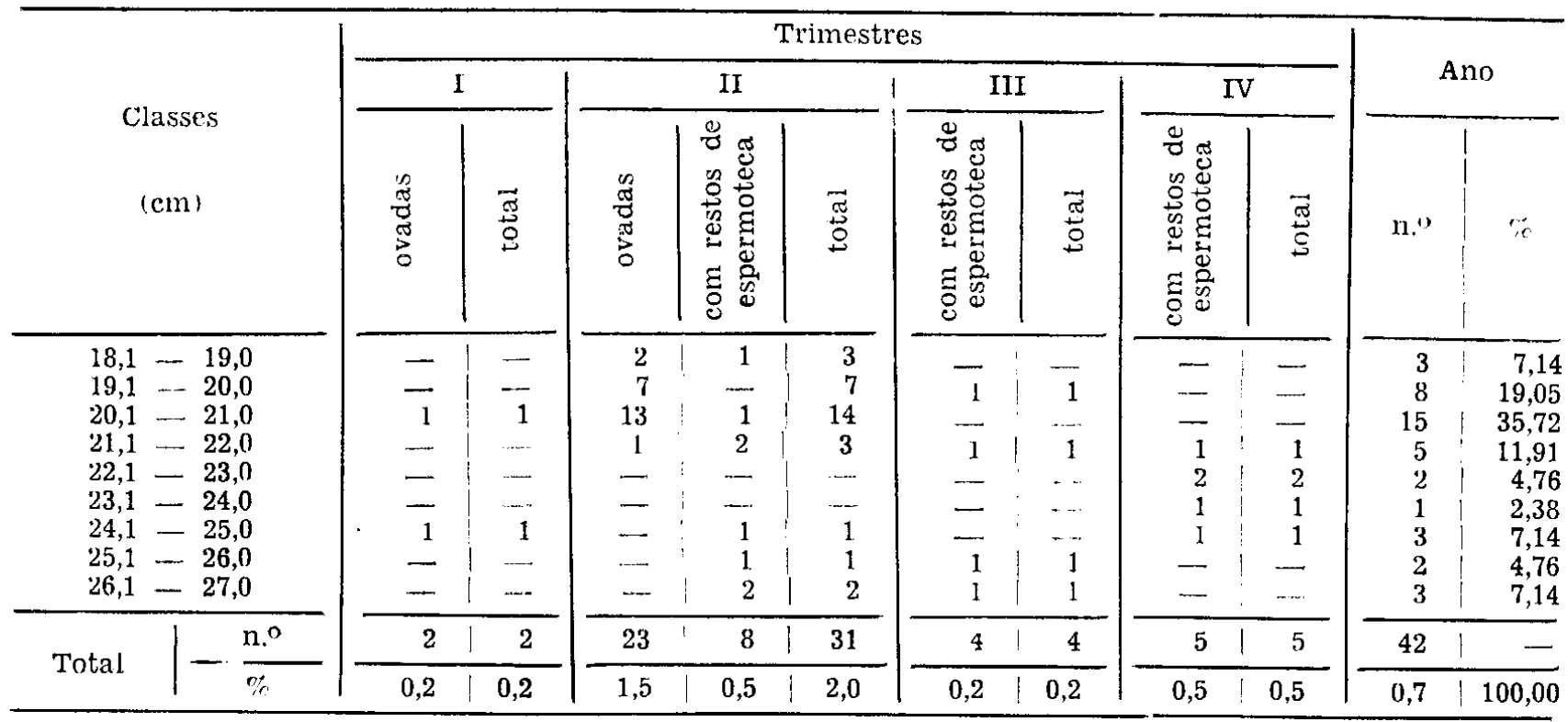

Observações: nāo foram amostradas fêmeas em reprodução nos meses de janeiro e fevereiro; foram omitidais as etapas do processo de reprodução não representadas nas amostras trimestrais; nas classes inferiores a $18,0 \mathrm{~cm}$ e superiores a $27,0 \mathrm{~cm}$ de comprimento total, não amostramos fêmeas em processo de repruüução. 


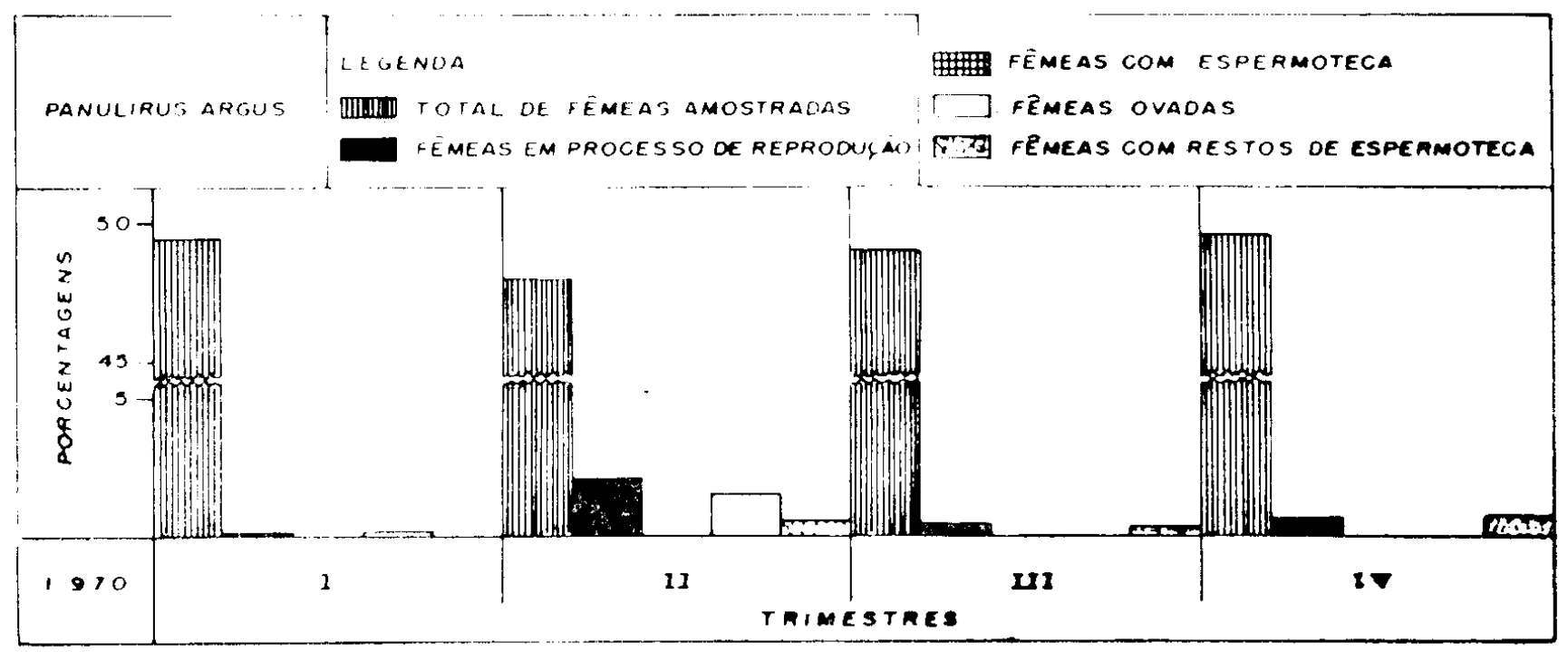

F'rgura 3 - Freqüências relativas de fêmeas em processo de reproduçāo, bem como em cada uma das suảs etapas, e o total das fêmeas amostradas, em relaçáo aos totais dos individuos amostrados da espécie $P a-$ nulirus argus (Latr.), em cada conjunto trimestral de amostras.

\section{T A B E L A V I}

Freqüencias de fèmeas de Panulirus laevicauda (Latr), capturadas em processo de reprodução, bem como em cada uma de suas etapas, por trimestres e classes de comprimento total, nas amostras de lagostas desembarcadas em Mucuripe (Fortaleza - Ceará - Brasil), durante o período de janeiro a dezembro de 1970 .

\begin{tabular}{|c|c|c|c|c|c|c|c|c|c|c|c|c|c|c|c|c|c|}
\hline \multirow[b]{3}{*}{$\begin{array}{c}\text { Classes } \\
(\mathrm{cm})\end{array}$} & \multicolumn{15}{|c|}{ Trimestres } & \multirow{2}{*}{\multicolumn{2}{|c|}{ Ano }} \\
\hline & \multicolumn{3}{|c|}{ I } & \multicolumn{4}{|c|}{ II } & \multicolumn{4}{|c|}{ III } & \multicolumn{4}{|c|}{ IV } & & \\
\hline & $\begin{array}{l}0 \\
0 \\
0 \\
0 \\
0 \\
0\end{array}$ & 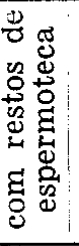 & $\begin{array}{l}\text { శే } \\
\text { 号 }\end{array}$ & 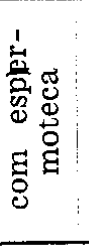 & 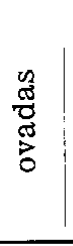 & 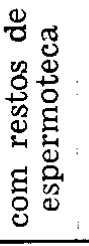 & 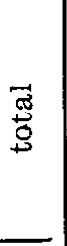 & 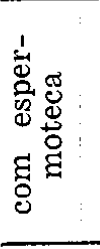 & 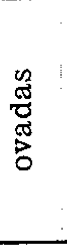 & 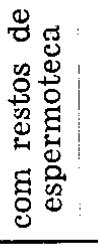 & $\begin{array}{l}\underset{\Phi}{\Phi} \\
\stackrel{\Phi}{\infty}\end{array}$ & 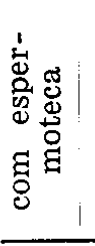 & 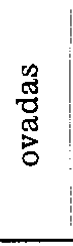 & 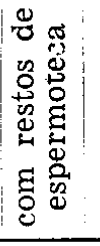 & 焉 & n. ${ }^{\circ}$ & $\%$ \\
\hline $14,1-15,0$ & 1 & -1 & 1 & - & 31 & 3 & 6 & $-!$ & -1 & -1 & - & -1 & -1 & -1 & - & 71 & 0,43 \\
\hline $15,1-16,0$ & 4 & -1 & 4 & $-{ }^{\prime}$ & 9 & 13 & 22 & -1 & 1 & 3 & 4 & -1 & 1 & -1 & 1 & 31 & 1,88 \\
\hline $16,1-17,0$ & 15 & 2 & 17 & $1:$ & 23 & 44 & 68 & $-{ }^{\prime}$ & 6 & 13 & 19 & 1 & 17 & 11 & 29 & 133 & 8,09 \\
\hline $17,1-18,0$ & 48 & 10 & 38 & $2 \mid$ & 60 & 113 & 175 & 3 & 14 & $51 !$ & 68 & $1:$ & 90 & 57 & 148 & 449 & 27,29 \\
\hline $18,1-19,0$ & 57 & 14 & 71 & -1 & 52 & 105 & 157 & 3 & 32 & 75 & 110 & 4 & 134 & 86 & 224 & 562 & 34,16 \\
\hline $19,1-20,0$ & 38 & 11 & 49 & 1 & 24 & 31 & 56 & 1 & 16 & 46 & 63 & 3 & 68 & 53 & 124 & 292 & 17,75 \\
\hline $20,1-21,0$ & 10 & 9 & 19 & $-\cdots !$ & 2 & $7 !$ & 9 & 1 & 10 & 17 & 28 & -- & 28 & 16 & 44 & 100 & 6,08 \\
\hline $21,1-22,0$ & 8 & 2 & 10 & -1 & 2 & 6 & 3 & 2 & 2 & 3 & 7 & 1 & 6 & 3 & 10 & 35 & 2,13 \\
\hline $22,1-23,0$ & 7 & 11 & 8 & -1 & 3 & 3 & 6 & $-i$ & 1 & 6 & 7 & $-\cdots$ & 1 & 2 & 3 & 24 & 1,46 \\
\hline $23,1-24,0$ & 2 & -1 & 2 & -1 & 1 & $-!$ & 1 & - & 1 & $-!$ & 1 & -1 & 2 & 1 & 3 & 7 & 0,43 \\
\hline $24,1-25,0$ & -1 & - & - & -1 & -1 & -1 & - & -1 & -1 & $-!$ & $\cdots$ & $-:$ & - & 2 & 2 & 2 & 0,12 \\
\hline $25,1-26,0$ & 1 & 1 & 2 & -1 & -1 & -1 & - & -1 & -1 & $-!$ & - & -1 & - & -1 & - & 2 & 0,12 \\
\hline $26,1-27,0$ & -1 & -1 & 一 & -1 & $-!$ & -1 & - & -1 & - & $-!$ & -- & -1 & 一 & - & - & - & - \\
\hline $27,1-28,0$ & -1 & -1 & - & -1 & $1 !$ & -1 & 1 & -1 & -1 & -1 & - & -1 & -1 & -1 & -- & $1 !$ & 0,06 \\
\hline \multirow{2}{*}{ Total } & 191 & $50 !$ & 241 & $4^{1}$ & $180 !$ & 325 & 509 & $10 \mid$ & 83 & $214 !$ & 307 & 101 & 347 & 231 & 588 & 1.645 & - \\
\hline & 25,3 & 6,6 & 31,9 & 0,3 & $14,7 \mid$ & 26,5 & 41,5 & $0,9 \mid$ & $7,7 \mid$ & 19,8 & 28,4 & 0,6 & 20,7 & 13,8 & 35,0 & 34,7 & 100,00 \\
\hline
\end{tabular}

Observaçöes: foram omitidas as etapas do processu de reprodução não representadas nas amostras trimestrais; nas classes inferiores a $14.0 \mathrm{~cm}$ e superiores a $28,0 \mathrm{~cm}$ de comprimento total, não amostramos fêmeás em processo de reprodução.

pécie Panulirus argus e estatisticamente significante na espécie Panulirus laevicauda. Na espécie Panulirus argus, num total global de 5.759 indivíduos amostrados, os machos representaram $51,03 \%$ e as fêmeas $48,97 \%$. Já para a espécie Panulirus laevicauda, num total global de 4.741 indivíduos amostrados, os machos representaram $54,67 \%$ e as fêmeas $45,33 \%$. 


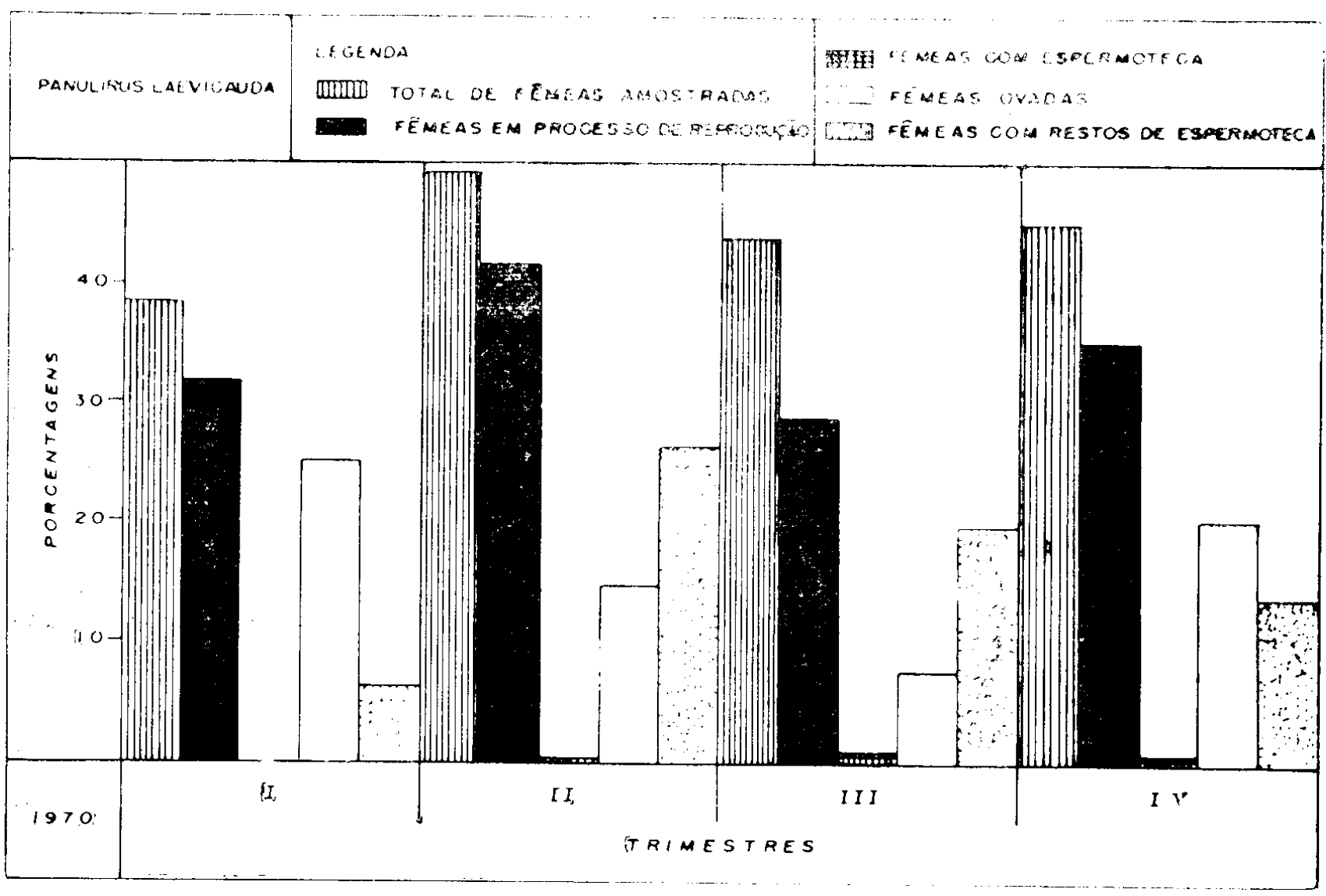

Figura 4 - Freqüencias relativas de fèmeas em processo de reprodução, bem como em cada uma das suä etapas, e o total das fêmeas amostradas, em relação aos totais dos indivíduos amostrados da espécie $P(t-$ nulirus laevicauda (Latr.), em cada conjunto trimestral de amostras.

Tomando em consideração os totais trimestrais amostrados de indivíduos da espécie Panulirus argus, verificamos que estatisticamente não houve diferente participação dos sexos nas amostragens.

Com respeito aos totais trimestrais amostrados de indivíduos da espécie Panulirus laevicauda, vemos que no primeiro, terceiro e quarto trimestres, houve diferente participação dos sexos, estatisticamente significante e com predomínio dos machos; no segundo trimestre também verificou-se diferente participação dos sexos, embora estatisticamente in- significante, com predomínio dos machos (tabela III ; figura 1) .

Mais uma vez se confirma que as fêmeas da espécie Panulirus argus são menos protegidas contra a ação de aparelhos de pesca do tipo manzuá, do que as da espécie Panulirus lacvicauda.

Os totais globais de machos e feemeas do ambas as espécies estudadas, distribuídos por classes de comprimento total (tabela II e III ; figura 2), mostram que a pesca agiu diferentemente sobre os sexos somente quanto à espécie Panulirus laevicauda.

T A B E L A V I I

Indices de densidade relativa de lagostas na area de Fortaleza (Ceara ... Brasil), duante o periodis de janeiro a dezembro de 1970 .

\begin{tabular}{|c|c|c|c|c|c|}
\hline \multirow{2}{*}{ Trimestres } & \multirow{2}{*}{$\begin{array}{c}\text { Esforgo } \\
\text { controlado } \\
\text { (manzuás/dia) }\end{array}$} & \multirow{2}{*}{$\begin{array}{l}\text { Lagostas } \\
\text { capturadas }\end{array}$} & \multicolumn{3}{|c|}{ Lagostas capturadas por manzuá/dia } \\
\hline & & & Panulirus argus & Panulirus laevicauda & Total \\
\hline $\begin{array}{l}\text { I } \\
\text { II } \\
\text { III } \\
\text { IV }\end{array}$ & $\begin{array}{r}14.755 \\
8.250 \\
6.930 \\
13.770\end{array}$ & $\begin{array}{r}24.258 \\
17.241 \\
6.746 \\
9.232\end{array}$ & $\begin{array}{l}1,57 \\
2.08 \\
0.92 \\
0,44\end{array}$ & $\begin{array}{l}0,07 \\
0,00 \\
0,05 \\
0,23\end{array}$ & $\begin{array}{l}1,64 \\
2,08 \\
0,97 \\
0,67\end{array}$ \\
\hline Total & 43.705 & 57.477 & 1,21 & 0,10 & 1,31 \\
\hline
\end{tabular}




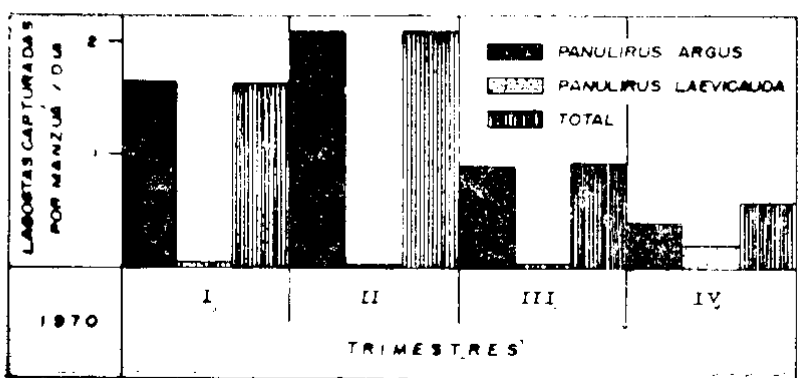

Figura 5 - Indices trimestrais da abundância relativa de lagostas das espécies Panulirus argus (Latr.) e Panulirus laevicauda (Latr.), na área de pesca em frente ao municipio de Fortaleza, durante o aso de 1970

Com respeito à esqécie Panulirus argus, verificamos que os indivíduos capturados tiveram comprimentos totais que variaram de 12,0 a $35,0 \mathrm{~cm}$.

Os indivíduos capturados da espécie $P a$ nulirus laevicauda tiveram comprimentos totais que variaram de 12,0 a $28,0 \mathrm{~cm}$; entre 15,0 e $19,0 \mathrm{~cm}$ de comprimento total, houve predomínio dos machos nas capturas.

A distribuição do total amostrado dos indivíduos da espécie Panulirus argus, por sexos e classes de comprimento total, mostra que tanto os machos como as fêmeas das classes compreendidas entre 17,0 e $22,0 \mathrm{~cm}$ foram os mais atingidos pela pesca, com máximo entre 19,0 e $20,0 \mathrm{~cm}$ de comprimento total (tabela II ; figura 2) .

A distribuição do total amostrado dos indivíduos da espécie Panulirus laevicauda, por sexos e classes de comprimento total, mostra que machos e fêmeas das classes compreendidas entre 16,0 e $20,0 \mathrm{~cm}$ foram os mais atingidos pela pesca, com máximo entre 18,0 e $19,0 \mathrm{~cm}$ de comprimento total (tabela III ; figura 2)

Para ambas as espécies estudadas, foram registrados indivíduos em processo de muda (tabela IV). As frequiencias absolutas anotadas não expressam com clareza a intensidade do processo na natureza. Para a espécie Panulirus argus, registramos indivíduos em processo de muda em todos trimestres, enquanto que para a espécie Panulirus laevicauda, somente no segundo e quarto trimestres.

Fêmeas de Panulirus argus, em processo de reprodução, foram registradas em todos os trimestres; esboçou-se um período de mais intensa reprodução no segundo trimestre (tabela $\mathrm{V}$; figura 3 ). Estas fêmeas se distribuíram entre 18,0 e $27,0 \mathrm{~cm}$ de comprimento total, havendo concentração entre 19,0 e $22,0 \mathrm{~cm}$, com máximo entre 20,0 e $21,0 \mathrm{~cm}$ de comprimento total (tabela $\mathrm{V}$ ) .

Em todos os trimestres considerados, registramos fêmeas de Panulirus laevicauda em processo de reprodução, sendo que no primeiro trimestre o fenômeno biológico se realizou com maior intensidade na população, esboçando-se um outro período de reprodução, com menor intensidade, no último trimestre (tabela VI ; figura 4). Estas fêmeas se distribuíram nas classes compreendidas entre 14,0 e $28,0 \mathrm{~cm}$ de comprimento total, havendo concentração entre 17,0 e $20,0 \mathrm{~cm}$, com máximo entre 18,0 e $19,0 \mathrm{~cm}$ de comprimento total (tabela VI) .

Os dados mostram que a captura de fêmeas jovens, de ambas as espécies, foram muito baixas. O mesmo aconteceu com as fêmeas de espermoteca íntegra. Para a espécie Panulirus argus, as freqüências de fêmeas ovadas, quando estas ocorreram, foram muito baixas; para a espécie Panulirus laevicauda, as freqüências de fêmeas ovadas foram bem elevadas no primeiro e último trimestres do ano considerado.

Os índices de densidade relativa, calculados para cada espécie, são bem diferentes daqueles apresentados em anos anteriores. E que os dados serviram de suporte para a determinação dos índices de densidade relativa, presentemente considerados, são aqueles pertinentes aos mapas de bordo, envolvendo substancialmente pescarias realizadas por embarcações da frota industrial, que normalmente operam em pesqueiros mais distantes da costa.

Para a espécie Panulirus argus, os índices trimestrais de densidade relativa, em ordem decrescente, se distribuíram da seguinte maneira: segundo, primeiro, terceiro e quarto trimiestres (tabela VII ; figura 5) .

Para a espécie Panulirus laevicauda, os índices trimestrais de densidade relativa, em ordem decrescente, se distribuíram da seguinte maneira: quarto, primeiro, terceiro e segundo trimestres (tabela VII ; figura 5) .

$\mathrm{Na}$ área estudada, a menor abundância de lagostas correspondeu ao último trimestre, e a maior se verificou no segundo trimestre (tabeì VII) .

O índice anual de densidade relativa se mostrou praticamente igual ao do ano anterior, e inferior aos registrados para os anos de 1964 a 1968 (na espécie Panulirus argus o índice registrado foi inferior aos dos anos de 1964,1965 e 1967 , e superior aos dos anos de 1966 , 1968 e 1969 ; na espécie Panulirus laevicauda o índice registrado foi inferior aos dos anos de 1964 a 1969).

\section{SUMMARY}

With this paper the Marine Sciences Laboratory of the Federal University of Ceará carries on a serial study on the spiny lobster fishery biology in the State of Ceará (Brazil).

The material, on which this study is supported, was caught off Fortaleza County, and landed at Mucuripe Beach. It corresponds 
to samples of 210 days of fishing, carried out during the period from January 2nd to December 30th, 1970 , by motor boats operating with traps baited chiefly with fishes. A total of 10,500 individuals was sampled. For the knowledge of the spiny lobsters relative density, the industrial fleet fishing $\log$ data in the same area and period, were also utilized.

Only the species Panulirus argus (Latr.) and Panulirus laevicauda (Latr.) were present in the samples; the first represented by $54.8 \%$ and the latter by $45.2 \%$ of the total samplings. Among 5,759 individuals sampled of the first species, $51.03 \%$ were males and $48.97 \%$ females. Among 4,741 individuals sampled of the second species, $54.67 \%$ were males and $45.33 \%$ females.

In the samples of the species Panulirus argus, males never predominated over females throughout the year.

Among the individuals of the species $\mathrm{Pa}$ nulirus laevicauda, males predominated over females during the year, except for the second trimester where no significant difference was observed.

Females of the species Panulirus argus are less protected against the action of the fishing gears of the trap type than those of the species Panulirus laevicauda.

In the species Panulirus argus the individuals sampled varied from 12.0 to $35.0 \mathrm{~cm}$ of total length. As to the individuals sampled of the species Panulirus laevicauda they varied from 12.0 to $28.0 \mathrm{~cm}$ of total length.

Females of the species Panulirus argus in reproduction process were reported in all trimesters; a supposed period of more intensive reproduction was observed in the second trimester. These females were distributed from 18.0 to $27.0 \mathrm{~cm}$ of total length.

Females of the species Panulirus laevicauda in reproduction process were reported in all trimesters considered, and the periods of more intensive reproduction were the first and the fourth trimesters. These females were distributed from 14.0 to $28.0 \mathrm{~cm}$ of total length.

Catches of young females of both species, in general were low. As to egged females of the species Panulirus argus, their frequencies in the catches, in the trimesters when they occurred, were also low. For the species Panu- lirus laevicauda, the frequencies of females bearing sperm-sac were low; in general, the frequencies of egged females were high, principally during the first and fourth trimesters.

In the area studied, the least abundance of spiny lobsters corresponded to the fourth trimester, and the greatest abundance to the second trimester.

The trimestral indexes of relative density, when studied for each species, showed that the species Panulirus argus occurred most abundantly in the second trimester, and the least abundance in the fourth trimester; while the species Panulirus laevicauda was most abundant in the fourth trimester, and least abundant in the second trimester.

Indexes of relative density referring to 1970 were practically the same as those recorded for 1969 , and lower than those recorded for the yearly periods from 1964 to 1968 .

\section{REFERÊNCIAS BIBLIOGRÁFICAS}

Paiva, M. P. \& Costa, R. S. - 1963 - Estudos de biologia da pesca de lagostas no Ceará - Dados de 1962 . Arq. Est. Biol. Mar. Univ. Ceará, Fortaleza, 3 (1) : 27-52, 7 figs.

Paiva, M. P. \& Costa, R. S. - 1964 - Estudos de biologia da pesca de lagostas no Ceará - Dados de 1963. Arq. Est. Biol. Mar. Univ. Ceará, Fortaleza, 4 (2) : $45-70,7$ figs.

Paiva, M. P. \& Costa, R. S. - 1965 - Estudos de biologia da pesca de lagostas no Ceará - Dados de 1964. Arq. Est. Biol. Mar. Univ. Ceará, Fortaleza, 5 (2) : 127-150, 9 figs.

Paiva, M. P. \& Costa, R. S. - 1966 - Estudos de biologia da pesca de lagostas no Ceará - Dados de 1965. Arq. Est. Biol. Mar. Univ. Fed. Ceará, Fortaleza, 6 (2) : 167-193, 9 figs.

Paiva, M. P. \& Costa, R. S. - 1967 - Estudos de biologia da pesca de lagostas no Ceará - Dados de 1966. Arq. Est. Biol. Mar. Univ. Fed. Ceará, Fortaleza, 7 (2): 147-171, 9 figs.

Paiva, M. P. \& Costa, R. S. - 1968 - Estudos de biologia da pesca de lagostas no Ceará - - Dados de 1967. Arq. Est. Biol. Mar. Univ. Fed. Ceará, Fortaleza, 8 (2) : 187-210, 9 figs.

Paiva, M. P. \& Costa, R. S. - 1969 - Estudos de biologia da pesca de lagostas no Ceará — Dados de 1968. Arq. Ciên. Mar, Fortaleza, 9 (2) : 133-146, 5 figs.

Paiva, M. P. \& Costa, R. S. - 1970 - Estudos de biologia da pesca de lagostas no Ceará - Dados de 1969. Arq. Ciên. Mar, Fortaleza, 10 (2): 131-142, 5 figs.

Paiva, M. P. \& Silva, A. B. - 1962 - Estudos de biolegia da pesca de lagostas no Ceará - Dados de 1961. Arq. Est. Biol. Mar. Univ. Ceará, Fortaleza, 2 (2) : 21-24, 7 figs. 